\title{
Estudio de los flujos de dispersión de los residuos plásticos en el Golfo de Cádiz debido a las corrientes superficiales marinas: una propuesta didáctica para iniciar a los alumnos de $1^{\circ}$ ESO en la indagación científica escolar
}

\author{
José María Torres Castillo \\ C.D.P.Argantonio.Cádiz. España.jm.torres@colegioargantonio.net \\ ORCID: https:// orcid.org/0000-0002-3112-2786
}

[Recibido: 21 Octubre 2018. Revisado: 12 Marzo 2019. Aceptado: 25 Abril 2019]

\begin{abstract}
Resumen: La contaminación del medio marino por plásticos es una amenaza medioambiental emergente que comenzó a notificarse en los años setenta del siglo pasado, y que en la actualidad afecta a la mayoría de organismos marinos. En el ámbito educativo, rara vez se aborda de forma práctica el estudio de las corrientes marinas y su capacidad de transporte de los residuos plásticos a zonas alejadas de donde se produjeron, de manera que la percepción que tienen los alumnos de este problema es muy baja. En este artículo se describe una experiencia de enseñanza-aprendizaje por investigación que, realizada con alumnos de 12 años, permitió descubrir algunas de las posibles trayectorias que siguen los residuos plásticos en el Golfo de Cádiz. Para ello se lanzaron, desde la playa de Santa María del Mar (Cádiz) y en tres fechas diferentes, un total de 21 botellas con mensajes. Gracias a la colaboración ciudadana, se pudieron reconstruir las posibles trayectorias de 5 de estas botellas. Dos de ellas fueron transportadas por las corrientes hacia el noroeste y alcanzaron las playas del Algarve. Otras dos fueron llevadas hacia el sureste y vararon en las playas de Conil y Tarifa respectivamente. Finalmente, la quinta fue encontrada cerca de la playa de lanzamiento. La evaluación final de la experiencia sugiere que los alumnos ampliaron y modificaron sus conocimientos sobre las corrientes marinas y el problema medioambiental de los residuos plásticos en el medio marino y fueron capaces de generar datos científico-escolares sobre el tema investigado.
\end{abstract}

Palabras clave: Residuos plásticos. Corrientes superficiales marinas. Golfo de Cádiz. Enseñanza-aprendizaje por investigación.

Study of the dispersion flows of plastic debris in the Gulf of Cádiz (Spain) due to marine surface currents: a didactic proposal to initiate the students of 1st ESO in scholarly scientific inquiry

\begin{abstract}
The contamination of the marine environment by plastics is an emerging environmental threat that began to be notified in the seventies of the last century, and that currently affects the majority of marine organisms. In the educational field, the study of marine currents and their capacity to transport plastic debris to areas far from where they were produced is rarely addressed in a practical way, so that the perception that students have of this problem is very low. In this article we describe a teaching-learning experience by research that, with 12-year-old students, allowed us to discover some of the possible trajectories of plastic debris in the Gulf of Cadiz. For this purpose, a total of 21 bottles with messages were launched from Santa María del Mar beach (Cadiz) and on three different dates. Thanks to the citizen collaboration, the possible trajectories of 5 of these bottles could be reconstructed. Two of them were transported by currents to the northwest and reached the beaches of Portugal. Two others were taken to the Southeast and beached on the beaches of Conil and Tarifa respectively. Finally, the fifth was found on the same launch beach. The final evaluation of the experience suggests that the students expanded and modified their knowledge about marine currents and the environmental problem of plastic debris in the marine environment and were able to generate scientific-school data on the subject researched.
\end{abstract}

Keywords: Plastic debris. Marine surface currents. Gulf of Cadiz. Teaching-learning by research.

Para citar este artículo: Torres Castillo J. M. (2019) Estudio de los flujos de dispersión de los residuos plásticos en el Golfo de Cádiz debido a las corrientes superficiales marinas: una propuesta didáctica para iniciar a los alumnos de $1^{\circ}$ ESO en la indagación científica escolar. Revista Eureka sobre Enseñanza y Divulgación de las Ciencias 16(3), 3501. doi: 10.25267/Rev_Eureka_ensen_divulg_cienc.2019.v16.i3.3501 


\section{Introducción}

Los grandes avances alcanzados en el conocimiento científico desde finales del siglo pasado y las aplicaciones tecnológicas que de él se han derivado, están cambiando a gran velocidad la vida de las personas. Esta veloz transformación de nuestras sociedades industriales hacia los patrones de la sociedad del conocimiento, plantea nuevas exigencias de adaptación a nuestros sistemas educativos (Esteve 2003), haciéndose imprescindible una alfabetización científica y tecnológica como parte esencial de la educación de todos los ciudadanos (Furió y Vilches 1997, Jenkins 1999, Furió et al. 2001, Acevedo 2004). Actualmente, según Sadler y Zeidler (2009), el modelo de alfabetización científica y tecnológica al que se tiende es aquél que incorpora los componentes de situaciones reales que afectan a la ciudadanía, y en las que la ciencia y la tecnología se ven involucradas. De esta manera, todos los ciudadanos deben ser capaces de enfrentarse a dilemas relacionados con la ciencia (OCDE 2016), resolviendo situaciones de la vida cotidiana de forma análoga a como se actúa ante los problemas de las actividades científicas y tecnológicas (MEC 2015b). Uno de esos inquietantes retos a los que se enfrenta la ciencia en nuestros días, generado paradójicamente por el desarrollo de la propia tecnología y convertido en un problema medioambiental emergente, es el de la contaminación de los océanos por los residuos plásticos (Derraik 2002, Moore 2008, Lebreton et al. 2018).

Aunque el primer polímero completamente sintético, la bakelita, se fabricó en 1909 (García, 2009), no es hasta principios de los años 50 del siglo pasado cuando comienza la producción de plásticos a gran escala (Waring et al. 2018). A principios de los años setenta aparecen los primeros informes de residuos plásticos en el medio ambiente marino y, en la actualidad, constituyen ya el principal componente de la basura marina (Bergmann et al. 2015). Quizá la amenaza medioambiental más evidente debida a la existencia de plásticos en los océanos, consiste en el impacto negativo que, para la conservación de las poblaciones de especies marinas, suponen las muertes por ingesta de plásticos o enmallamiento. En este sentido, se ha constatado que ingieren plásticos el $50 \%$ de las especies de aves marinas, las siete especies de tortugas existentes y el $66 \%$ de los mamíferos marinos, detectándose cada vez más en peces e invertebrados (Khün et al. 2015). Por otro lado, las estimaciones sobre la cantidad de organismos que sucumben cada año enredados en las redes de pesca abandonadas la sitúan en millones de individuos (Moore 2008). Además, los plásticos son sensibles a la luz ultravioleta, y gran parte de ellos se fragmentan y degradadan en las playas y en la superficie del mar convirtiéndose en microplásticos ( $<5 \mathrm{~mm}$ ) (Moore 2008, Andrady 2011, Galloway 2015) y nanoplásticos $(<100 \mathrm{~nm})$ (Mattsson 2018). Estas micro y nanopartículas se desplazan en la columna de agua y alcanzan los sedimentos del fondo marino (Galgani et al. 2015), donde numerosas especies zooplanctónicas los ingieren (Andrady 2011, Desforges 2015), entrando en las redes tróficas (Eriksen et al. 2014, Ryan 2015). Se ha constatado la presencia de microplásticos y nanoplásticos en especies de peces y mariscos de interés comercial (Thompson 2015, Waring et al. 2018), que de este modo entran en la cadena alimentaria humana (Waring et al. 2018), pudiendo causar alteraciones en los cromosomas que conducen a la infertilidad, la obesidad y el cáncer (Sharma y Chatterjee 2017).

Resulta difícil calcular la cantidad de plástico presente actualmente en el medio marino, aunque recientes estimaciones la sitúan en 5,25 billones de partículas, que corresponden a un peso de 268.940 toneladas (Eriksen et al. 2014). De igual forma, resulta muy complejo determinar los movimientos y concentraciones de las partículas de plástico en los océanos, pues estos flujos varían ampliamente debido a la proximidad a los núcleos urbanos, los usos costeros, los vientos y las corrientes marinas (Galgani 2015). Se ha constatado que los vientos y las corrientes superficiales marinas redistribuyen los plásticos a través de los océanos (Eriksen et al. 2014), viajando largas distancias antes de varar (Ryan et al. 2009, Galgani 2015) o 
de acumularse en medio de las cuencas oceánicas debido a los giros oceánicos (Kazarian 2006, Meléndez y Meléndez 2013). Como resultado, los residuos plásticos son ahora contaminantes ubicuos incluso en las áreas más remotas del mundo (Barnes et al. 2009).

A escala local, los flujos de residuos plásticos en el Golfo de Cádiz pueden ser más difíciles de identificar si cabe, pues se trata de una de las áreas más complejas de circulación marina del mundo, al constituir la zona de intercambio con el mar Mediterráneo a través del Estrecho de Gibraltar (Peliz et al. 2007). Para mayor complejidad, la circulación superficial está dominada por dos remolinos ciclónicos, limitados al sur por un chorro de agua que entra en el Golfo de Cádiz y que se mueve hacia el este (García-Lafuente et aı. 2006). Se ha determinado además, que los vientos intensos de la zona provocan la aparición de nuevas corrientes superficiales en su entorno (Seoane 1965), formando ángulos que varían entre los $13^{\circ}$ y los $38^{\circ}$ a la derecha de estos vientos (Martínez et al. 1998). Pero lo que parece indiscutible es que los microplásticos han entrado ya en las redes tróficas del Golfo de Cádiz, pues se ha detectado en una especie demersal de interés comercial de la zona como el cazón moteado, Scyliorbinus canicula (Bellas et al. 2016).

Frente a este desafío global y debido a la ausencia de datos geoespaciales básicos y cuantitativos de los flujos oceánicos de los residuos plásticos, en los próximos años, según Bergmann et al. (2015), se hará necesaria la participación ciudadana para monitorizar este tipo de contaminación. Por lo tanto, se presenta la oportunidad de que la escuela participe activamente colaborando en la recogida de información sobre la basura marina, algo necesario a escala local (Eastman et al. 2014). Además su participación puede permitir que los escolares tomen conciencia sobre un problema medioambiental de gran complejidad de estudio y de consecuencias difícilmente observables de forma directa en el medio, lo que inicialmente les lleva a no percibirlo como un problema grave. Flores (2004) propone que la aproximación de los alumnos al estudio de procesos complejos se puede llevar a cabo a partir de elementos cotidianos, lo que facilita la construcción conceptual y su comprensión. En este sentido, en la experiencia que aquí se presenta, se diseñó un sencillo estudio científico-escolar sobre los flujos de los residuos plásticos en el mar, utilizando una técnica que en el pasado suministró abundante información científica sobre las corrientes marinas. Esta técnica consiste en abandonar a la deriva botellas selladas con instrucciones, para que algún colaborador fortuito las recoja y las devuelva al laboratorio, indicando el lugar y el momento de su hallazgo (Strahler 1986).

Hasta ahora, y desde la educación ambiental, el acercamiento al problema de los plásticos se ha abordado a partir de la participación de escolares en estudios de reconocimiento visual y análisis de los residuos hallados durante prospecciones en la playa, tal y como han propuesto Artolachipi y Calvo (2006) o Eastman et al. (2014). La presente experiencia didáctica propone, sin embargo, una aproximación diferente al problema de la basura en el medio marino, basada en el estudio científico-escolar de las corrientes marinas como principal variable que causa su dispersión en el océano (Eriksen et al. 2014). Con la ayuda de ciudadanos que encontraron las botellas fortuitamente y que informaron de sus hallazgos a través del correo electrónico, los escolares reconstruyeron las rutas seguidas por estas botellas en el Golfo de Cádiz.

\section{Características de la propuesta didáctica}

En los últimos años, y desde la enseñanza de las Ciencias, se han desarrollado en nuestro país propuestas de enseñanza-aprendizaje dirigidas a promover la génesis del conocimiento científico-escolar a partir de la realización de investigaciones, lo que se considera hoy día una de las líneas más fructíferas y debatidas en Didáctica de las Ciencias (Franco-Mariscal 2015). La relevancia de estas investigaciones escolares radica en su capacidad de promover el logro de 
aprendizajes con elevados niveles de significatividad, integración y funcionalidad (Cañal 2012). No obstante, estas propuestas basadas en la investigación escolar siguen siendo aún minoritarias $y$, aunque se reconoce la importancia que tiene la formación científica, ésta ha recibido una atención muy reducida en la Educación Secundaria (Pedrinaci 2012). Sigue siendo, por lo tanto, poco habitual la realización de trabajos de investigación en el contexto escolar de la etapa obligatoria (Franco-Mariscal 2015), aún cuando los proyectos de investigación en equipo se encuentran dentro del currículo básico de la Educación Secundaria Obligatoria (MEC 2015a).

En este contexto, la presente propuesta didáctica se basó en el diseño y puesta en práctica de una investigación escolar que persiguió, por un lado, que los escolares tomaran conciencia del grave daño que este tipo de basura antropogénica genera en los ecosistemas marinos. Y, por otro lado, darles a conocer como se puede generar conocimiento científico-escolar sobre un problema medioambiental. La experiencia didáctica se desarrolló como parte de la asignatura Biología-Geología de $1^{\circ}$ ESO, durante dos cursos escolares 2016/17 y 2017/18, y contó con la participación de tres grupos de 31 alumnos de $1^{\circ}$ ESO (12 años) del centro educativo Colegio Argantonio (Cádiz) ; un grupo en el primer año académico y dos en el siguiente. En este artículo se describe la experiencia didáctica implementada en el curso 2017/18, y que contó con los resultados previos obtenidos durante el curso anterior.

Como estrategias metodológicas se utilizaron, por un lado, un enfoque constructivista del aprendizaje, orientado hacia la actividad del alumno. Y, por otro lado, la metodología del trabajo experimental adaptada a los escolares. Los resultados de este trabajo fueron divulgados a la comunidad educativa del Centro a través de una serie de conferencias que utilizaron materiales digitales elaborados por los propios alumnos. La acción del docente se centró en la presentación del problema medioambiental y su relación con las corrientes marinas, y en la coordinación del trabajo mediante la orientación y seguimiento de los alumnos en su ejecución, favoreciendo la reflexión y evaluando su aprendizaje.

\section{Objetivos de la propuesta didáctica}

1. Descubrir mediante la indagación científica la existencia de corrientes marinas en el Golfo de Cádiz y su relación con la dispersión de los residuos que se generan en la costa.

2. Introducir a los alumnos en la metodología del trabajo científico, en un contexto real y análogo al que desarrollan los científicos, de manera que les sirva para comprender como se construye la Ciencia.

3. Aprender a divulgar los resultados de una investigación científico-escolar a través de conferencias, elaborando para ello el material digital apropiado.

4. Reconocer el grave problema medioambiental que suponen los plásticos en el medio marino y promover actitudes responsables en el uso de los plásticos que eviten su llegada al mar.

\section{Descripción y desarrollo de la experiencia didáctica}

\section{Primera sesión. Actividad inicial motivadora y planteamiento del problema medioambiental}

La literatura constituye un instrumento que permite aprender aprovechando la necesidad inherente al ser humano de contar y escuchar historias, sirve para llamar la atención del alumnado y aumentar su motivación, y establece cauces para mejorar la comprensión de conceptos (Del Pino et al. 2017). Además, el uso de textos literarios en los procesos de enseñanza, permite abordar la realidad como un todo cuyas partes se interrelacionan de 
manera compleja (Algarra 2013), lo que da más sentido a lo que los alumnos estudian. Por todo ello, se decidió llevar a cabo la lectura de un texto literario como actividad inicial motivadora, concretamente el primer capítulo de la obra de Julio Verne Los hijos del capitán Grant en América del Sur. En dicho capítulo, se describe la captura de un tiburón martillo en aguas del Canal del Norte por los marineros del Duncan, y su posterior sacrificio en la cubierta del barco. Tras una inspección de los intestinos del escualo, los marineros descubren una botella con un mensaje en su interior. En el texto, se sugiere que el capitán Grant introdujo un mensaje de auxilio en la botella, con la esperanza de que las corrientes marinas la llevasen hasta alguien que le pudiera socorrer. Pero en su camino por el océano, se interpuso la voracidad del tiburón.

A continuación, se pidió a los escolares que respondieran a un cuestionario basado tanto en la lectura como en sus experiencias personales. Este cuestionario inicial sirvió como guión para un coloquio desarrollado posteriormente, en el que se indagaron sus conocimientos previos sobre las corrientes marinas, los residuos plásticos y las posibles relaciones entre ambos conceptos. Las preguntas fueron las siguientes: 1. ¿Has observado los restos de organismos y objetos que el mar deposita en la orilla de la playa tras los temporales? 2. ¿Con qué intención arrojó el capitán Grant la botella con el mensaje al mar? 3. Mientras te bañas en la playa, ¿has experimentado la fuerza de alguna corriente marina? 4. ¿Cómo pudo llegar la botella a los intestinos del tiburón? 5. ¿Sabes qué son las corrientes marinas y las causas que las generan? 6. ¿Conoces alguna relación entre las corrientes marinas y la basura generada por el hombre? 7. ¿Has oído hablar de los giros oceánicos subtropicales y de su relación el transporte de plásticos en el medio marino? 8. ¿Has oído hablar de los micro y nanoplásticos? 9. ¿Conoces las consecuencias que tiene para la fauna marina la existencia de plásticos en los océanos?. Las cinco últimas preguntas se volvieron a pasar a los alumnos al final de la secuencia didáctica, con la finalidad de comprobar los avances alcanzados en el conocimiento de esos conceptos.

Durante el coloquio posterior a la lectura y al cuestionario, se propuso a los escolares relacionar el viaje de la botella del capitán Grant, con la existencia de corrientes marinas y su capacidad para transportar la basura a través del océano. Algo que se vio reforzado con la experiencia que muchos de ellos poseían de la llegada a la orilla de la playa de organismos tales como medusas, algas y, cómo no, de basura de origen humano. Así como por haber experimentado la pérdida de flotadores o pelotas, mar adentro, en días de fuerte viento de levante. El descubrimiento de una botella de vidrio, realizado por los marineros del Duncan en los intestinos del tiburón, dio pie a comentar la interacción entre la fauna marina y la basura generada por los humanos, y sus posibles consecuencias. Fue el momento de introducir en el diálogo la existencia y persistencia de los residuos plásticos en el medio marino, debido a sus escasas características de biodegradabilidad, su transformación en microplásticos y sus consecuencias para la fauna. Además, y aunque no era uno de los objetivos del estudio, la lectura incitó a discutir los encontrados sentimientos de atracción por su belleza y, al mismo tiempo de terror, que los tiburones inspiran en el ser humano. Así como su desigual relación con el hombre, como se aprecia en el relato. Para finalizar esta primera sesión, y como consecuencia de los interrogantes planteados en las cuatro últimas preguntas del cuestionario, se utilizó la pantalla digital para visualizar mapas de corrientes marinas, e imágenes y noticias existentes en la red sobre la acumulación de plástico en los giros oceánicos y los efectos de los plásticos sobre la fauna marina. Estas imágenes causaron un gran impacto en los alumnos y prepararon el escenario para proponer la investigación científico-escolar. 


\section{Segunda sesión. Paso 1 de la investigación científico-escolar}

A partir de la fascinación que produjo en los escolares la historia contada a través de la lectura y el impacto de las imágenes visualizadas en la red, el docente les propuso llevar a cabo una investigación para descubrir la existencia de corrientes marinas en el entorno cercano al colegio y su capacidad para transportar residuos plásticos. Para ello, se utilizaron botellas selladas con mensajes tal y como se describió en el texto literario.

La investigación escolar se dividió en los siguientes pasos: 1. Planteamiento del problema y objetivo de la investigación; 2. Formulación de las hipótesis previas; 3. Metodología, planteamiento y diseño de la experimentación; 4. Registro y procesamiento de los datos; 5. Análisis de los datos y conclusiones.

Paso 1. Planteamiento del problema y objetivo de la investigación.

En este paso, los alumnos mostraron su interés por la problemática a tratar, a través del planteamiento de las siguientes preguntas científicas: ¿existen corrientes marinas en las costas gaditanas?. Y, si existen, ¿en qué direcciones se desplazan?. ¿Serán capaces de arrastrar con ellas la basura marina?. Y, si es así, ¿hacia dónde? y ¿qué consecuencias puede acarrear al medio ambiente?.

Con la finalidad de acotar los objetivos de la investigación, el profesor facilitó a los alumnos información básica sobre los factores que influyen en la generación de las corrientes marinas superficiales en la zona de estudio. De esta manera, se informó de forma básica sobre el marco teórico y el estado del conocimiento de las corrientes marinas en el Golfo de Cádiz. A continuación, divididos en grupos de cinco alumnos, discutieron sobre la formulación del objetivo de la investigación. Tras una puesta en común en la que intervinieron los vocales de cada grupo y el docente, se definió por consenso el siguiente objetivo: describir espacial y temporalmente los flujos de dispersión de los residuos plásticos en el Golfo de Cádiz.

\section{Tercera sesión. Pasos 2 y 3 de la investigación científico-escolar}

Paso 2. Formulación de las hipótesis previas.

A partir de las experiencias previas de los alumnos, y con la ayuda del docente y de la información científica facilitada por éste, los alumnos formularon las siguientes hipótesis de trabajo:

- Las corrientes superficiales marinas de la costa atlántica de la provincia de Cádiz se generan principalmente por los vientos dominantes de levante y poniente.

- Debido a la dirección de los vientos dominantes y a la forma de la costa, existirán dos corrientes opuestas que desplazarán los residuos plásticos hacia el Sureste y hacia el Noroeste respectivamente.

Paso 3. Metodología, planteamiento y diseño de la experimentación.

Tras una nueva puesta en común, primero en pequeños grupos y luego en gran grupo, los alumnos y el docente fijaron la experimentación en los pasos que se describen a continuación:

a. La metodología utilizada sería de tipo Lagrangiano (Strahler 1986, Font 1987), que consiste en el uso de botellas selladas con mensajes. Las botellas serían de vidrio, preferentemente no coloreado, reutilizadas para este segundo uso y selladas con tapón de corcho.

b. Se lanzarían 7 botellas, una por cada grupo de cuatro alumnos formado para la fase de experimentación. Los lanzamientos se efectuarían coincidiendo con la hora de clase de la asignatura y desde el extremo del espigón Sur de la playa $\mathrm{St}^{\mathrm{a}} \mathrm{M}^{\mathrm{a}}$ del Mar (Cádiz), el más cercano al colegio. 
c. El experimento se replicaría en primavera e invierno.

d. El mensaje indicaría que la botella era parte de una investigación sobre corrientes y basura marinas en las costas de Cádiz, realizada por alumnos de $1^{\circ}$ ESO del colegio Argantonio (Cádiz). Se le añadiría además el lugar y la fecha de lanzamiento, junto con la petición de colaboración a aquella persona que la encontrara, que debería informar por correo electrónico del lugar y fecha de su localización. Esto último implicó la participación ciudadana en esta fase del estudio, sin la cual no se habrían obtenido resultados. Para centralizar toda la información recibida, se decidió incluir en el mensaje la dirección de correo electrónico del profesor.

e. Se utilizó la página web de la AEMet para registrar las variables "velocidad del viento" $(\mathrm{km} / \mathrm{h})$, "dirección del viento" en Cádiz, en el momento de cada lanzamiento.

Cuarta sesión. Continuación del paso 3 de la investigación científico-escolar: preparación de las botellas y lanzamiento.

En esta sesión se llevaron a cabo la elaboración del mensaje, escrito a mano y sobre papel, la introducción en el interior de la botella y su sellado con el tapón de corcho (figura 1). Algunos grupos quemaron los bordes del mensaje para darle un aspecto antiguo y un toque romántico a la experimentación. Se lanzaron un total de 21 botellas, 7 en cada cada una de las siguientes fechas de lanzamiento: 06/04/2017, 14/12/2017 y 19/12/2017 (figura 2).

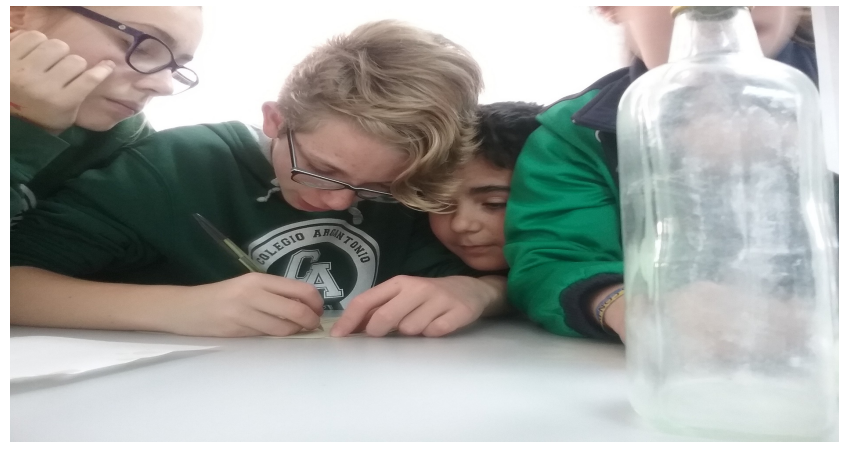

Figura 1. Preparación de los mensajes para introducirlos en las botellas.

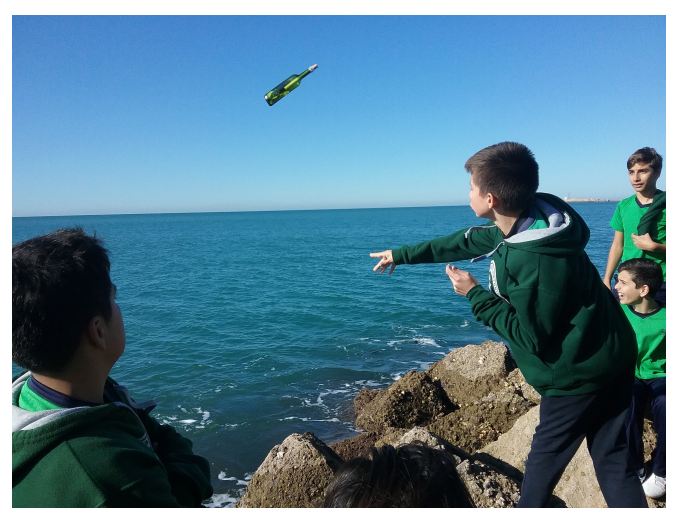

Figura 2. Lanzamiento de una de las botellas en la playa de Santa María del Mar (Cádiz).

Quinta y sexta sesiones. Paso 4 de la investigación científico-escolar: registro y procesamiento de los datos

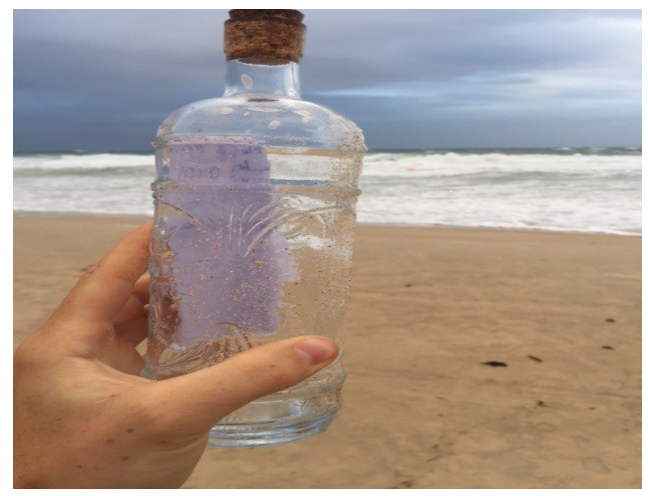

Figura 3. Botella encontrada por un observador el 27 de diciembre de 2017 en la playa de Los Lances (Tarifa).

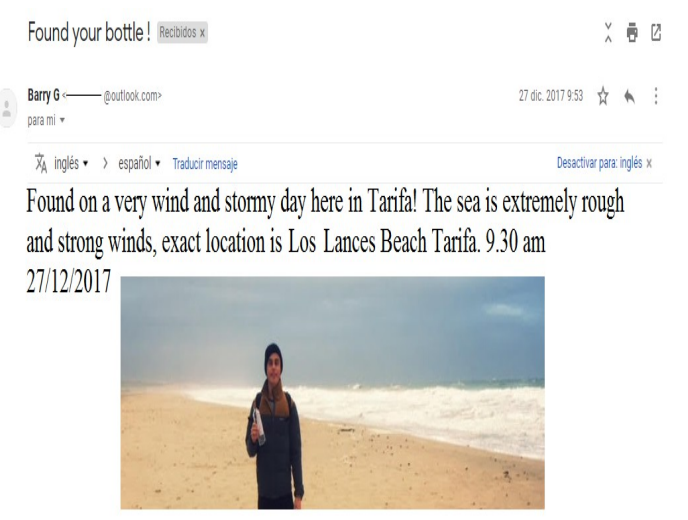

Figura 4. Correo electrónico recibido de un observador, informando del hallazgo de una de las botellas. 
Las sesiones quinta y sexta no tuvieron continuidad en el tiempo con las anteriores, pues se debió esperar a recibir información del hallazgo de las botellas. De las 21 botellas que se lanzaron, se recibieron noticias de la recuperación de 5 de ellas, lo que supuso el $24 \%$ del total de botellas utilizadas en la experimentación (figuras 3 y 4).

Los lugares y fechas de lanzamiento y recuperación aparecen ordenados en la tabla 1, elaborada por los alumnos.

Tabla 1. Registro de los datos obtenidos en la experimentación. Localidad de lanzamiento: Playa de Santa María del Mar (Cádiz-España).

\begin{tabular}{|l|l|l|}
\hline BOTELLAS & $\begin{array}{l}\text { FECHAS DE LANZAMIENTOS } \\
\text { Y DIRECCIONES DEL VIENTO }\end{array}$ & $\begin{array}{l}\text { FECHAS DE LOCALIZACIÓN, LOCALIDADES } \\
\text { DE VARAMIENTO Y DISTANCIAS } \\
\text { RECORRIDAS }\end{array}$ \\
\hline Botella 1 & $\begin{array}{l}06 / 04 / 2017 \\
\text { ESE }\end{array}$ & $\begin{array}{l}20 / 04 / 2017 \\
\text { Praia do Barril (Tavira-Portugal) } \\
137 \mathrm{~km} \text {; Vmedia mínima: } 9,8 \mathrm{~km} / \text { día }\end{array}$ \\
\hline Botella 2 & $\begin{array}{l}\text { ESE } / 04 / 2017 \\
\text { Botella 3 }\end{array}$ & $\begin{array}{l}\text { 14/12/2017 } / 04 / 2017 \\
\text { Praia do Barril (Tavira-Portugal) } \\
137 \mathrm{~km}\end{array}$ \\
\hline Botella 4 & $\begin{array}{l}19 / 12 / 2017 \\
\text { ONO calma }\end{array}$ & $\begin{array}{l}14 / 12 / 2017 \\
\text { Playa de Santa María del Mar (Cádiz-España) } \\
100 \mathrm{~m}\end{array}$ \\
\hline Botella 5 & $\begin{array}{l}19 / 12 / 2017 \\
\text { ONO }\end{array}$ & $\begin{array}{l}\text { Playa de Castilnovo (Conil de la Fra.-España) } \\
32 \mathrm{~km} \text {; Vmedia mínima: } 4 \mathrm{~km} / \text { día }\end{array}$ \\
\hline
\end{tabular}

Para una mejor interpretación de los datos obtenidos, éstos se representaron gráficamente en un mapa en papel donde se dibujaron las posibles trayectorias seguidas por las botellas,

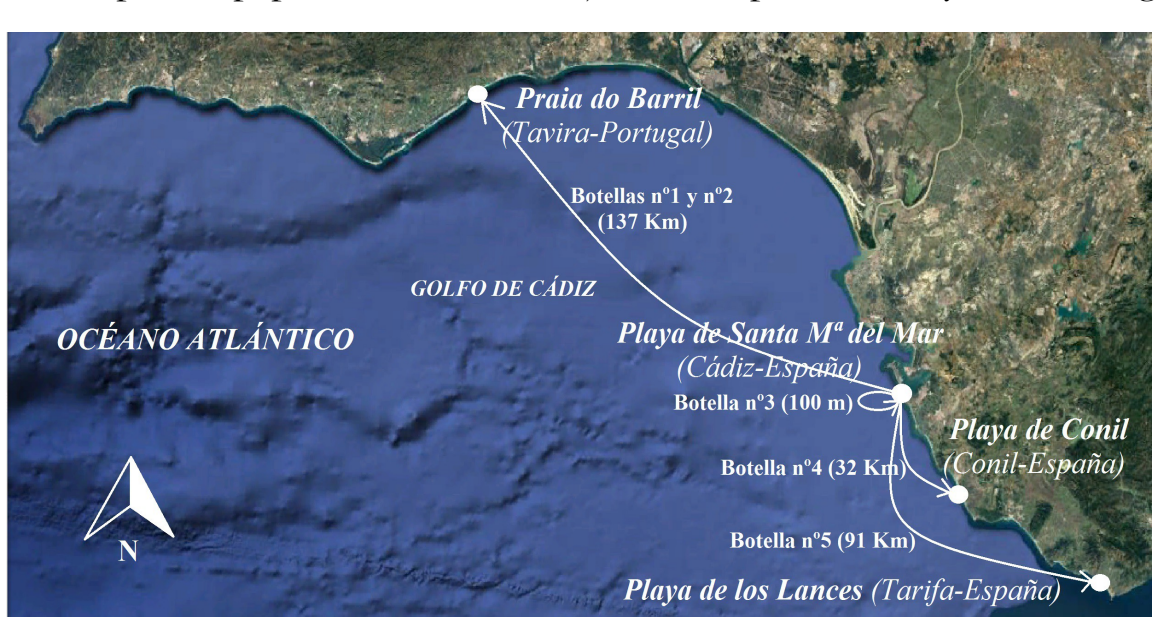

Figura 5. Representación gráfica de las trayectorias seguidas por las botellas en el Golfo de Cádiz. Elaboración de los alumnos. deducidas a partir de las localizaciones de lanzamiento y de recogida. Posteriormente, los mapas se digitalizaron utilizando diferentes programas de tratamientos de imágenes, según la elección de cada grupo de alumnos (figura 5). 


\section{Séptima y octava sesiones. Paso 5 de la investigación científico-escolar: análisis de los datos y conclusiones}

En este quinto paso se analizaron los datos obtenidos en la experimentación, se relacionaron con las posibles variables que intervinieron, especialmente lo vientos existentes en los momentos de los lanzamientos, y se elaboraron las conclusiones. Todo ello supuso un gran esfuerzo colectivo, a partir de la discusión y puesta en común de los pequeños avances que cada grupo fue alcanzado por separado. En esta fase del trabajo se ejercitó la capacidad crítica y reflexiva de los alumnos, para poder llevar a cabo una evaluación y una interpretación coherentes de los datos. Así, en una primera aproximación al análisis de los datos, el docente advirtió a los alumnos sobre la fiabilidad de las interpretaciones que se podían extraer de los datos obtenidos. Los escolares debieron diferenciar lo que se podía afirmar, de lo que sólo era una conjetura o una aproximación. De esta manera, se concluyó que la fecha en la que los observadores encontraron las botellas no tuvo por qué ser obligatoriamente la fecha de llegada de éstas a las playas. Las botellas pudieron haber llegado antes de que los observadores las encontraran. Por lo tanto, la duración de la navegación calculada a partir de las fechas de lanzamiento y de localización sólo podía ser aproximada, y no servía como dato objetivo para determinar la velocidad media de la corriente. Al contrario, los lugares donde los observadores las localizaron constituyeron unos datos más útiles, pues sirvieron para describir los posibles recorridos realizados por las botellas. No obstante, también la trayectorias propuestas fueron sólo aproximaciones y simplificaciones de la realidad. Al mismo tiempo, se discutió con los alumnos algunas de las limitaciones de esta metodología, pues sobre un problema científico complejo como el transporte de objetos en el mar, inciden un conjunto de variables cuyos efectos son difíciles de separar. Por ejemplo, con la metodología utilizada no se pudo aislar el efecto directo del viento sobre las botellas, ni el de las corrientes de marea. Fue ésta una fase donde afloraron las diferencias existentes en las capacidades de análisis y deducción de los componentes de cada grupo. Pero, sin duda, el trabajo cooperativo, con el intercambio de puntos de vista y de opinión, enriqueció a todos ellos y sirvió, especialmente, para ayudar en la reflexión a aquellos con un menor grado madurativo de pensamiento formal. Este fue el caso, por ejemplo, de la representación gráfica de las rutas seguidas por las botellas. Pues, en un primer momento, algunos de los alumnos trazaron líneas rectas para representar las trayectorias seguidas por las botellas. Lo que en el caso de la botella encontrada en la Playa de Los Lances, y debido a la forma de la costa, una trayectoria de este tipo entre Cádiz y Tarifa obligaba a que parte de ella se dibujara por tierra firme, lo cual era inviable. Sólo la discusión y comparación con las trayectorias trazadas por sus compañeros, les sacó del error y les hizo ver la posibilidad de una trayectoria curva por mar. Por lo tanto, el resultado final de dichas representaciones gráficas fue mejorado tras el trabajo cooperativo. Finalmente y como resultado del trabajo de análisis, interpretación y discusión, se llegaron a las siguientes conclusiones finales, que dieron respuesta al estudio escolar de la dispersión de los residuos plásticos en el Golfo de Cádiz, y que confirmaron sus hipótesis iniciales: «Los residuos plásticos generados en la ciudad se dispersan a lo largo de toda la costa del Golfo de Cádiz debido a las corrientes superficiales marinas. En invierno, una corriente que fluye en dirección ESE, desplaza los residuos generados en la capital a las playas del Sur de la provincia. En primavera, una corriente que se desplaza en dirección ONO, transporta los residuos plásticos más de un centenar de kilómetros hasta hacerlos varar en las playas del Algarve, lo que se convierte en un tipo de contaminación transfronteriza». 


\section{Novena, décima y undécima sesiones. Comunicación de la investigación y de los resultados}

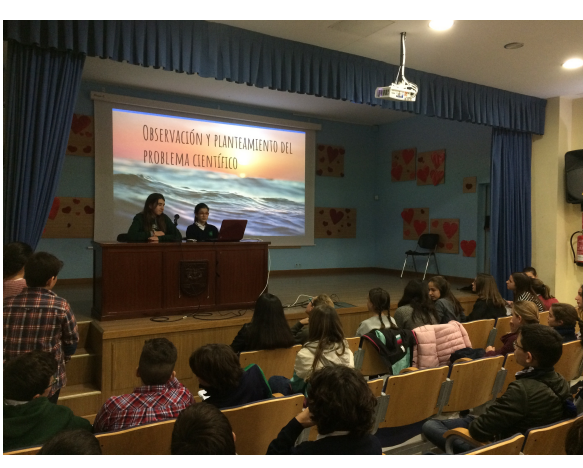

Figura 6. Conferencia para los alumnos del Centro, celebrada el 8 de junio, Día Mundial de los Océanos.
En esta última fase de la experiencia didáctica se discutió con los alumnos la importancia de dar a conocer los resultados de la investigación, tanto a la comunidad escolar como al público en general, de manera que sus conclusiones pudieran revertir en un cambio de actitud y comportamiento respecto a la generación de la basura plástica en la ciudad. En el presente trabajo, este importante aspecto consistió en una serie de charlas a la comunidad educativa del colegio (alumnos y padres) con la utilización de las TICs, que sirvieron además para concienciar sobre el problema medioambiental que suponen los plásticos en el océano. Por eso, una de las fechas elegidas fue el 8 de junio, Día Mundial de los nuestros Océanos" (figura 6). Se realizaron dos presentaciones digitales, una por cada curso, en las que participaron todos los alumnos. Cada una de las dos presentaciones digitales se dividió en las siguientes partes: observaciones y planteamiento del problema; elaboración de las hipótesis previas; fase de experimentación: metodología y lanzamiento de las botellas; tablas de datos y representación gráfica; análisis e interpretación de los resultados; acumulación de plástico en los giros oceánicos, microplásticos y consecuencias sobre los ecosistemas marinos; medidas para evitar la contaminación plástica del mar.

\section{Evaluación de la experiencia didáctica}

\section{Mediante instrumentos de evaluación}

Para evaluar la presente experiencia didáctica, se tuvieron en cuenta tanto las producciones finales como los procesos de aprendizaje de los escolares. De esta manera, se recopilaron evidencias de sus aprendizajes en diversos contextos a lo largo de todo el desarrollo de la experiencia, a través de las siguientes estrategias: observación del trabajo individual y en grupo en las diferentes etapas del trabajo; entrevistas del docente con cada alumno y con cada uno de los grupos; valoración de las producciones, en papel (cuaderno de trabajo e informes finales), en formato digital (resultados de las búsquedas en la red, correos electrónicos, elaboración de tablas, mapas y presentaciones digitales), y en las exposiciones orales y conferencias en público.

\section{Mediante cuestionarios inicial y final de la secuencia didáctica}

A partir de las cinco últimas preguntas del cuestionario inicial, se pudo comprobar el desconocimiento general que los escolares poseían de los conceptos clave al inicio de la experiencia. Así, en la encuesta inicial, el $95 \%$ de los escolares no supo explicar correctamente el concepto de corriente marina. De éstos el $21 \%$ lo confundieron con las mareas. Al finalizar la secuencia didáctica el $100 \%$ de los alumnos la definió correctamente y señaló al viento como principal causa de la existencia de las corrientes superficiales, tal y como se había deducido en la investigación escolar. Al inicio de la experiencia, el $92 \%$ no había oído hablar de la acumulación de plástico en los giros oceánicos y ninguno ellos de los microplásticos. Y al finalizar ésta, los alumnos utilizaron correctamente imágenes de estos dos conceptos para la elaboración de las presentaciones digitales. Y, por supuesto, ningún alumno intuyó en el cuestionario inicial el transporte de los plásticos por las corrientes marinas. Algo que quedó 
finalmente demostrado con la investigación escolar. Por otro lado, el $94 \%$ de los escolares ya conocía, antes de comenzar la experiencia, los dos impactos más visibles que los residuos plásticos generan sobre la fauna marina, la ingestión y el enmallamiento. Se pudo averiguar, que la fuente de esta información partía principalmente de la acción divulgativa de los medios de comunicación, especialmente sensibilizados en los últimos tiempos.

Al final de la experiencia se les pasó a los alumnos un segundo cuestionario anónimo con el objetivo, en este caso, de conocer su grado de satisfacción con la experiencia didáctica y el cambio de actitud frente al problema medioambiental estudiado. Las preguntas y las respuestas emitidas por los alumnos junto a sus porcentajes, se presentan a continuación:

1. Valora de 0 a 10 esta experiencia de aprendizaje mediante investigación. Puntuación media: 9,7. 2. ¿Qué parte del trabajo te ha gustado más? Respuesta más repetida: lanzamiento de las botellas desde el espigón de la playa (95\%); 3. ¿Qué parte del trabajo te ha resultado más difícil? Respuesta más repetida: ponerse de acuerdo en el trabajo en equipo (95\%); 4. ¿Te gustaría realizar otro trabajo científico? Sí: $100 \%$; ¿Q ¿ué puedes hacer para contribuir a solucionar el problema de la contaminación marina por plásticos? Respuestas con mayores porcentajes: Evitar arrojar plásticos a la playa: $92 \%$. Colocar carteles informativos en las playas para evitar que se abandonen residuos plásticos: $82 \%$. Realizar campañas de recogida de plásticos en la playa: $75 \%$.

\section{Discusión y conclusiones de la propuesta didáctica}

En este artículo se describe una experiencia didáctica llevada a cabo con 62 alumnos de 12 años (nivel $1^{\circ} \mathrm{ESO}$ ), para estudiar el problema medioambiental de los residuos plásticos en los océanos e iniciarlos en el trabajo científico. Por lo tanto, el docente aceptó el reto de llevar a cabo una propuesta didáctica que incluyó a la totalidad del alumnado, con toda su diversidad en capacidades e intereses, poniéndolos en contacto con la ciencia e iniciando así su alfabetización científica.

El enfoque singular de esta propuesta didáctica consistió en diseñar y poner en práctica un sencillo estudio científico-escolar, que permitió a los alumnos descubrir flujos de dispersión en el Golfo de Cádiz de la basura plástica generada en su ciudad. De este modo, se consiguió que los escolares visualizaran las corrientes marinas y las entendieran como la principal causa de la dispersión de los plásticos en los océanos. El trabajo dio pie además, a que los escolares investigaran en la red sobre la transformación de los plásticos en microplásticos y su entrada en las redes tróficas de los ecosistemas marinos, reflexionando sobre el destino y las consecuencias generadas por los residuos abandonados en el litoral. Por lo tanto, un problema medioambiental de gran complejidad, se abordó través de un estudio técnicamente asequible para los escolares, aprovechando el entorno marino cercano al colegio. A este respecto, hay que hacer notar el exiguo tratamiento que recibe la contaminación del medio marino en el currículo de $1^{\circ} \mathrm{ESO}$, por lo que la presente propuesta didáctica vino a cubrir el hueco existente en esta temática.

En lo que respecta a los resultados oceanográficos obtenidos en la investigación escolar, el análisis de los datos obtenidos a partir de las botellas recuperadas, permitió describir dos corrientes superficiales diferentes en las fechas de experimentación. La primera de estas corrientes, con dirección ONO, se describió a partir de las botellas encalladas en Tavira (Portugal) en abril de 2017. Este flujo coincide con el esquema de circulación ciclónica de primavera-verano propuesto por García-Lafuente et al. (2006). El segundo flujo, opuesto al anterior y con dirección ESE, se dedujo a partir de las botellas varadas en las playas del Sur de la provincia de Cádiz en diciembre de ese mismo año. Este flujo coincide con la corriente de finales de otoño y principios de invierno detectada por Criado-Aldeanueva et al. (2009), que se 
desplaza hacia el Sureste reemplazando a la célula ciclónica situada sobre la plataforma continental oriental del Golfo de Cádiz. Estos resultados vendrían a confirmar que la circulación superficial sobre la plataforma continental está sujeta a cambios de flujo estacionales (Criado-Aldeanueva et al. 2009) y evidencian la correlación entre el viento zonal y la circulación superficial (Criado-Aldeanueva et al. 2006), que era una de las hipótesis iniciales del trabajo. Así, la circulación anticiclónica parece estar asociada con los vientos del oeste durante la mayor parte del año, mientras que las inversiones de flujo tienen lugar bajo episodios del este (Criado-Aldeanueva et al. 2009), lo que se corresponde con la segunda hipótesis del trabajo. De todo ello se deduce la complejidad del proceso de dispersión de los residuos plásticos en el Golfo de Cádiz, dependiente de la estacionalidad y de los vientos existentes en cada momento.

En el plano puramente didáctico, y una vez comenzada la investigación, la mayor dificultad que se presentó fue conseguir que los escolares trabajaran en equipo coordinadamente, algo que los propios alumnos resaltaron en el encuesta final. Aunque la percepción de los alumnos indica que este aspecto no fue logrado, la observación del desarrollo de la secuencia didáctica y los resultados finales del trabajo indican que, cuando menos, sí se realizaron ciertos progresos. Indudablemente, se requieren muchas más horas de trabajo en equipo para que los escolares asimilen el trabajo cooperativo.

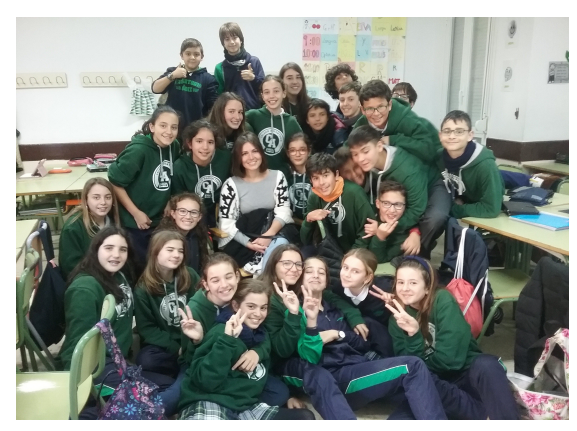

La evaluación de las cinco preguntas que se repitieron en los cuestionarios inicial y final, indicaron un significativo avance en el conocimiento de la acumulación de basura en lo giros oceánicos y los microplásticos. Asimismo, y en el actual contexto pedagógico europeo, con las competencias clave como el elemento central del currículo, la experiencia pedagógica descrita en este artículo permitió que los alumnos trabajaran al mismo tiempo un conjunto de competencias. De este modo, la competencia científica se abordó a través de la enseñanza-aprendizaje por Figura 7. Visita al aula de una de las investigación, con el objetivo de acercar a los estudiantes al personas que localizaron las botellas. contexto en el que trabajan los científicos (Franco-Mariscal 2015), y lograr aprendizajes significativos y funcionales para el desenvolvimiento en el medio (Cañal 1999). Así, los alumnos practicaron en equipo la formulación de hipótesis, el diseño y puesta en práctica de un experimento, el tratamiento matemático y gráfico de los resultados, la emisión de unas conclusiones y, finalmente, la comunicación de los resultados mediante charlas divulgativas. Además, aplicaron el análisis y el razonamiento para discutir e interpretar los resultados, compararlos y contrastarlos con lo que ya se conocía sobre el problema de estudio, para así poder corroborar las hipótesis iniciales. Además, los alumnos trabajaron la competencia digital al utilizar las tecnologías de la información y la comunicación para la búsqueda y selección de información científica relacionada con el tema, para responder mediante e-mails a las personas que localizaron las botellas, para la elaboración de los mapas digitales de las trayectorias y, finalmente, para elaborar las presentaciones digitales, que supusieron un importante proceso creativo. En cuanto a la comunicación lingǘstica, referida a la utilización del lenguaje como instrumento de comunicación oral y escrita (Bolívar 2008), se trabajó en contextos diferentes, tales como en la decodificación y comprensión de textos de carácter científico, en la discusión con los compañeros y con el docente en cada uno de los pasos de la investigación, en la redacción de los mensajes de las botellas, en la comunicación a través de castellano, inglés y alemán con las personas que encontraron las botellas, en la preparación de los textos para las presentaciones digitales y, finalmente, durante las conferencias. Un momento especial fue cuando una de las 
personas que localizaron las botellas vino al aula invitada por los alumnos y se le obsequió con una charla informativa de la experiencia (figura 7). La experiencia didáctica ofreció a los alumnos, además, la oportunidad de poner en práctica su autonomía intelectual mediante la búsqueda, comparación y selección de información, lo que es fundamental para el aprendizaje permanente y que depende de que se genere curiosidad y necesidad de aprender (MEC 2015b).

Finalmente, la concienciación medioambiental lograda en los escolares se pudo comprobar a través de las medidas que propusieron para paliar el problema del abandono de plásticos en las playas, y que fueron incluidas en las charlas divulgativas. La alta puntuación que dieron a la experiencia en la encuesta final, superando el 9,5 de media, y el hecho de que la totalidad de los alumnos mostrase su interés por realizar un nuevo trabajo científico, indicó su elevado grado de satisfacción con la experiencia didáctica. A este respecto, los escolares destacaron el momento de la visita a la playa para arrojar las botellas, como el paso de la experimentación que más les gustó. Lo que coincide con la opinión expresada por escolares de edades parecidas en otras experiencias científicas como la del Primer Muestreo Nacional de Microplásticos en las Playas de Chile (Hidalgo-Ruz et al. 2012). Y es que, como afirma Sancho (2006), aprender es algo emocional, por lo que la enseñanza debe dirigirse también a actividades materiales y prácticas, y no sólo a actividades mentales e intelectuales. Para concluir, y como propuesta de mejora de la experiencia, se baraja la posibilidad de que en el próximo curso se visite la Facultad de Ciencias del Mar de la Universidad de Cádiz, para poder conocer el trabajo de los científicos que investigan la teledetección de los plásticos en los océanos.

\section{Agradecimientos}

Deseamos expresar nuestro agradecimiento a Steffi y Klaus Jürgen Beer, Pedro Costa, Gloria Guillén Rosado, Matthias Daun y Barry John Grimes, quienes por azar encontraron las botellas, pero que se preocuparon de enviar los correos electrónicos que informaron de sus hallazgos, sin los cuales no se habrían obtenido resultados en esta investigación científico-escolar.

\section{Referencias}

Acevedo J. A. (2004) Reflexiones sobre las finalidades de la enseñanza de las ciencias: educación científica para la ciudadanía. Revista Eureka sobre Enseñanza y Divulgación de las Ciencias 1 (1) 3-16.

Algarra S. J. (2013) La literatura en la enseñanza de las ciencias sociales. Tinkuy: Boletín de investigación y debate 20, 23-55.

Andrady A. L. (2011) Microplastics in the marine environment. Marine Pollution Bulletin 62, 1596-1605.

Artolachipi A., Calvo J. (2006) El litoral de la provincia de Cádiæ. Manual para el profesor. Consejería de Medio Ambiente. Junta de Andalucía.

Barnes D. K. A., Galgani F., Thompson R. C., Barlaz M. (2009) Accumulation and fragmentation of plastic debris in global environments. Philosophical Transactions of the Royal Society B 364, 1985-1998.

Bellas J., Martínez-Armental J., Martínez-Cámara A., Besada V., Martínez-Gómez C. (2016) Ingestion of microplastics by demersal fish from the Spanish Atlantic and Mediterranean coasts. Marine Pollution Bulletin 109 (1), 55-60.

Bergmann M., Gutow L., Klages M. (eds.) (2015) Marine Anthropogenic Litter. Springer, Cham. 
Bolívar A. (2008) El discurso de las competencias en España: educación básica y educación superior. REDU: Revista de Docencia Universitaria. Monográfico 11, Formación centrada en competencias II.

Cañal P. (2012) ¿Cómo evaluar la competencia científica? Investigación en la Escuela 78 (monográfico), 5-17.

Criado-Aldeanueva F., García-Lafuente J., Vargas J.M., Del Río J., Sánchez A., Delgado J., Sánchez J. C. (2006) Wind induced variability of hydrographic features and water masses distribution in the Gulf of Cadiz (SW Iberia) from in situ data. Journal of Marine Systems 63 (3-4), 130-140.

Criado-Aldeanueva F., García-Lafuente J., Navarro G., Ruiz J. (2009) Seasonal and interannual variability of the surface circulation in the eastern Gulf of Cadiz (SW Iberia). Journal of Geophysical Research 114, C01011.

Del Pino A., García G., Campos M. (2017) La literatura como vehículo para el aprendizaje de la ciencia: el ciclo del agua. Revista Iberoamericana de Ciencia Tecnología y Sociedad 12 (35), 201-215.

Derraik J. G. B. (2002) The pollution of the marine environment by plastic debris: a review. Marine Pollution Bulletin 44, 842-852.

Desforges J. P., Galbraith M., Ross P. S. (2015) Ingestion of Microplastics by Zooplankton in the Northeast Pacific Ocean. Archives of Environmental Contamination and Toxicology 69 (3), 320-330.

Eastman L., Hidalgo-Ruz V., Macaya-Caquilpan V., Núñez P., Thiel M. (2014) The potential for young citizen scientist projects: a case study of Chilean schoolchildren collecting data on marine litter. Revista de Gestão Costeira Integrada / Journal of Integrated Coastal Zone Management 14 (4), 569-579.

Eriksen M., Lebreton L. C. M., Carson H. S., Thiel M., Moore C. J., Borerro J. C., Galgani F., Ryan P. G., Reisser J. (2014) Plastic Pollution in the World's Oceans: More than 5 Trillion Plastic Pieces Weighing over 250,000 Tons Afloat at Sea. PLoS ONE 9 (12), e111913.

Esteve J. M. (2003) La tercera Revolución Educativa. La Educación en la Sociedad del Conocimiento. Paidós. Barcelona.

Flores F. (2004) El cambio conceptual: interpretaciones, transformaciones y perspectivas. Educación Química 15 (3), 256-269.

Font (1987) Nuevos aspectos del estudio de la circulación marina en el Mediterráneo noroccidental. Informes Técnicos del Instituto de Investigaciones Pesqueras 141, 3-28.

Francisco-Mariscal A. J. (2015) Competencias científicas en la enseñanza y el aprendizaje por investigación. Un estudio de caso sobre corrosión de metales en secundaria. Enseñanza de las Ciencias 33 (2), 231-252.

Furió C., Vilches A. (1997) Las actitudes del alumnado hacia las ciencias y las relaciones ciencia, tecnología y sociedad, en Luis del Carmen (coord.). La enseñanza y el aprendizaje de las ciencias de la naturaleza en la educación secundaria. Horsori. Barcelona.

Galgani F., Hanke G., Maes T. (2015) Global Distribution, Composition and Abundance of Marine Litter. pp. 29-56 en Marine Anthropogenic Litter. Part I. Bergmann et al. (eds.). 
Galloway T. S. (2015) Micro- and Nano-plastics and Human Health. pp. 343-366 en Marine Anthropogenic Litter, Part IV. Socio-economic Implications of Marine Anthropogenic Litter. Bergmann et al. (eds.).

García S. (2009) Referencias históricas de evolución de los plásticos. Revista Iberoamericana de Polímeros 10 (1), 71-80

García-Lafuente J., Delgado J., Criado-Aldeanueva F., Bruno M., Del Río J., Vargas J. M. (2006) Water mass circulation on the continental shelf of the Gulf of Cádiz. Deep Sea Research Part II: Topical Studies in Oceanography 53 (11-13), 1182-1197.

Hidalgo-Ruz V., Macaya V., Eastman L., Thiel M. (2012) Muestreo Nacional de Microplásticos en las Playas de Chile.

Jenkins E. (1999) School science, citizenship and the public understanding of science. International Journal of Science Education 21, 703-710.

Kazarian U. (2006) Islands of Garbage Continue To Grow in Pacific. Sustainable Development Law ana Policy 7, 63-84.

Khün S., Bravo-Rebolledo E. L., Van Franeker J. A. (2015) Deleterious Effects of Litter on Marine Life. pp. 75-116 en Marine Anthropogenic Litter, Part II. Biological Implications of Marine Litter. Bergmann et al. (eds.).

Lebreton L., Slat B., Ferrari F., Sainte-Rose B., Aitken J., Marthouse R., Hajbane S., Cunsolo S., Schwarz A., Levivier A., Noble K., Debeljak P., Maral H., Schoeneich-Argent R., Brambini R., Reisser J. (2018) Evidence that the Great Pacific Garbage Patch is rapidly accumulating plastic. Scientific Reports 8, 4666.

Martínez M., Cotos J.M., Arias J., Tobar A. (1998) Cálculo de corrientes superficiales marinas a partir de imágenes térmicas NOAA y estimación de la influencia de los vientos en su aparición: aplicación al suroeste de la Península Ibérica. Revista de la Asociación Española de Teledetección 9, 5-15.

Mattsson K., Jocic S., Doverbratt I., Hansson L-A. (2018) Nanoplastics in the Aquatic Environment. In Microplastic Contamination in Aquatic Environments, Zeng E.Y. (ed.), Chapter 13, 379-399.

MEC (2015a) Ministerio de Educación y Ciencia, Real Decreto 1105/2014, de 26 de diciembre, por el que se establece el currículo básico de la Educación Secundaria Obligatoria y del Bachillerato (BOE núm. 3, 3 de enero de 2015).

MEC (2015b) Ministerio de Educación y Ciencia, Orden ECD/65/2015, de 21 de enero, por la que se describen las relaciones entre las competencias, los contenidos y los criterios de evaluación de la educación primaria, la Educación Secundaria Obligatoria y el Bachillerato (BOE núm. 25, 29 de enero de 2015).

Meléndez M. A., Meléndez P. I. (2013) Influencia de la circulación eólica y marítima en la formación de las islas de basura en el mudo. Ciencia y Sociedad 38 (4), 743-791.

Moore C. J. (2008) Synthetics polymers in the marine environment: A rapidly increasing, longterm threat. Environmental Research 108 (2), 131-139.

OCDE (2016) PIS A 2015 Resultados Clave.

Peliz A., Dubert J., Marchesiello P., Teles-Machado A. (2007) Surface circulation in the Gulf of Cadiz: Model and mean flow structure Journal of Geophysical Research 112, C11015. 
Pedrinaci E. (2012) Enseñanza de las ciencias de la Tierra. Revista de la Asociación Española para la Enseñanza de las Ciencias de la Tierra 20 (2), 133-140.

Ryan P. G., Moore J. M., Van Franeker J. A., Moloney C. L. (2009) Monitoring the abundance of plastic debris in the marine environment. Philosopbical Transactions of the Royal Society $B$ 364, 1999-2012.

Ryan P. G. (2015) A Brief History of Marine Litter Research. pp. 1-25 en Marine Anthropogenic Litter. Bergmann et al. (eds.).

Sadler T. D., Zeidler D. L. (2009) Scientific Literacy, PISA, and Socio-scientific. Discourse: Assessment for Progressive Aims of Science Education. Journal of Research on Science Teaching 46 (8), 909-921.

Sancho J. M. (2006) Aprender a los 15 años: factores que influyen en este proceso. Revista de Educación Extraordinario, 171-193.

Seoane J. (1965) Las algas de interés industrial de la costa de Cádiz. Publicaciones Técnicas de la Junta de Estudios de Pesca 4, 225-252.

Sharma S., Chatterjee S. (2017) Microplastic pollution, a threat to marine ecosystem and human health: a short review. Environmental Science and Pollution Research 24, 21530 21547.

Strahler A. N. (1986) Geografía Física. Ediciones Omega. Barcelona.

Thompson R. C. (2015) Microplastics in the Marine Environment: Sources, Consequences and Solutions. pp. 185-200 en Marine Anthropogenic Litter, Part III Microplastics. Bergmann et al. (eds.).

Waring R. H., Harris R. M., Mitchell S. C. (2018) Plastic contamination of the food chain: A threat to human health? Maturitas 115, 64-68. 